\title{
Numerical Study of Inclusion Removal in Steel Continuous Casting Mold Considering Interactions Between Bubbles and Inclusions
}

\author{
Qingrui LAI, ${ }^{1)}$ Zhiguo LUO, ${ }^{1) *}$ Qinfu HOU, ${ }^{2)}$ Tao ZHANG, ${ }^{1)}$ Xiaoai WANG ${ }^{3)}$ and Zongshu ZOU ${ }^{1)}$ \\ 1) School of Metallurgy, Northeastern University, Shenyang, Liaoning, 110819 China. \\ 2) Department of Chemical Engineering, Monash University, Clayton, VIC 3800 Australia. \\ 3) HBIS Company Limited, Shijiazhuang, Hebei, 050023 China.
}

(Received on May 2, 2018; accepted on July 9, 2018; J-STAGE Advance published date: September 10, 2018)

\begin{abstract}
Bubble coalescence-breakup and bubble-inclusion interaction models were implemented into EulerianLagrangian models of steel liquid flow and discrete particles transport, which was applied to examine the interaction and removal of discrete bubbles and inclusions during continuous casting. Bubble distribution affected by coalescence-breakup and its connection with the inclusion removal rate were analyzed. The inclusion removal rates at different initial bubble diameters and inclusion diameters were predicted. Larger inclusions have a higher removal rate and the predicted removal rates ranging from $14 \%$ to $30 \%$ agree well with industrial experiment measurements. It is also found that smaller bubbles have a higher capacity to remove inclusions and very small bubbles may cause more production defects when it is attached to inclusions. These results show that the developed model can reasonably predict the behaviors of discrete bubbles and inclusions and their interactions in molten steel. Such a model should be useful for the operation of steel continuous casting.
\end{abstract}

KEY WORDS: steel continuous casting; bubble-particle interaction; inclusion removal; bubble coalescence and breakup.

\section{Introduction}

Continuous casting is an important procedure in steel production and its quality control. In this process, Argon gas is usually injected to prevent nozzle clogging. Argon bubbles could coalescence or breakup into different sizes, significantly affecting steel quality. Large bubbles float upward and escape from the liquid steel surface. However, small bubbles follow the main stream of molten steel and could be entrapped by the solidifying shell, resulting in pin holes, blisters and other defects in steel products. ${ }^{1)}$ Although argon bubbles could capture inclusions, carry and remove them into the top slag, bubbles attached with inclusions may also be entrapped, which is particularly harmful to steel products. ${ }^{2)}$ Hence transport and entrapment of discrete bubbles and inclusions, the coalescence and breakup of bubbles and bubble-inclusion attachment are crucial factors affecting steel product quality, which have not been well understood.

There are mainly two groups of approaches to model discrete phases in multiphase flows: the Eulerian and the Lagrangian methods. By the Eulerian method, different phases are treated mathematically as penetrating continua, having their own but similar governing equations. Thus, macroscopic statistical quantities (such as number density of solid particles) can be obtained, but the information of dis-

\footnotetext{
* Corresponding author: E-mail: luozg@smm.neu.edu.cn DOI: https://doi.org/10.2355/isijinternational.ISIJINT-2018-319
}

crete elements is averaged out. ${ }^{3,4)}$ The Lagrangian method solves the equations of motion for each discrete element, providing location, velocity, path and other information of individual elements. Discrete Particle Method (DPM), one of the Lagrangian approaches, has been applied to simulate the transport and entrapment of bubbles or inclusions in continuous casting mold. ${ }^{1,5-8)}$ However, discrete phases were considered as mass points, and the interaction between discrete phases was neglected.

In actual continuous casting process, large bubbles float upward while small bubbles tend to move towards sidewall. The bubbles moving towards sidewall may be captured by the solidifying shell, leading to defects in steel products. Such motions and the coalescence and breakup of bubbles in submerged entry nozzle $(\mathrm{SEN})^{9-11)}$ or mold ${ }^{11-13)}$ were observed in recent water model experiments. However, most numerical simulation studies ${ }^{1,5,6,12,14)}$ of bubble flow in continuous casting mold have not taken the crucial factor, coalescence-breakup of bubbles, into consideration. Based on the Eulerian approach, population balance model was applied to investigate bubble size distribution in this process from a statistic viewpoint. ${ }^{13,15)}$ In such studies the change of bubble number density was estimated by corresponding kernel function and applied to bubble population balance equation as source terms. To solve population balance equation, bubbles were divided into several fixed groups according to their sizes. Based on the Lagrangian approach, particle-scale simulation of gas-liquid flow in this process 
were conducted, ${ }^{16)}$ considering coalescence, bounce and breakup of bubbles. But the study was limited to gas-liquid two-phase flow where the inclusions were not considered.

The attachment of solid particles to bubble have been conducted mainly in gas-stirred steelmaking process. ${ }^{17-21)}$ Such studies can be largely divided into two categories according to the treatment of inclusion transport in molten steel. In the first category, ${ }^{2,22-24)}$ inclusion transport was ignored, and inclusions were assumed to be uniformly distributed in the molten steel. The number of inclusions attached to bubbles was estimated according to the volume swept by bubbles and the attachment probability of inclusions to a bubble. However, inclusions in a mold tend to follow the stream of molten steel, which should not be ignored. To overcome this limitation, the second category of studies ${ }^{25-27)}$ was developed in steelmaking process by combining the Eulerian approach and the population balance model to investigate inclusion attachment to bubble from a statistic viewpoint. However, coalescence and breakup of bubbles were ignored. Moreover, these studies assume that once an inclusion is attached to a bubble, it will be removed, and the mass of bubble was not changed. In fact, the attached inclusion will move together with the bubble and the mass of the bubble will change, and some of them may be entrapped instead of being removed.

A comprehensive model is developed in this work to consider the interaction and removal of discrete phases in molten steel. It considers the transport and entrapment of discrete phases, the coalescence and breakup of bubbles, and bubble-inclusion attachment. It was first used to study the gas-liquid two-phase flow and the general features were illustrated. The connection between bubble distribution and inclusion removal was then discussed. Finally, the inclusion removal rates under different conditions were obtained and compared to experimental measurements.

\section{Model Description}

A modified two-way coupled Euler-Lagrange model is developed. The flow field of liquid steel is calculated through computational fluid dynamics method and force balance for each individual inclusion or bubble to simulate their transient transport. The effect of the discrete phases on the continuum is considered by incorporating the exchange of momentum as a source term in the momentum equation of continuous phase. The interaction between discrete particles is modeled via calculating distances between discrete particles along with criterions to determine their interactive behaviors.

\subsection{Governing Equations of Fluid Phase}

Mass and momentum conservation equations for incompressible fluid in multiphase flow are given by

$$
\begin{gathered}
\frac{\partial}{\partial t}\left(\alpha_{l} \rho_{l}\right)+\nabla \cdot\left(\alpha_{l} \rho_{l} \vec{v}_{l}\right)=0 \ldots \ldots . . . \\
\frac{\partial}{\partial t}\left(\alpha_{l} \rho_{l} \vec{v}_{l}\right)+\vec{v}_{l} \cdot \nabla\left(\alpha_{l} \rho_{l} \vec{v}_{l}\right) \\
=-\nabla p+\nabla \cdot\left[\alpha_{l}\left(\mu_{l}+\mu_{t}\right)\right] \nabla \vec{v}_{l}+\vec{F}_{k}
\end{gathered}
$$

where $\alpha_{l}$ is the liquid-phase volume fraction, $\rho_{l}$ is the liquidphase density, $\vec{v}_{l}$ is the fluid-phase average velocity, $p$ is pressure, $\vec{F}_{k}$ is source term for momentum exchange with the discrete phases, including drag force, buoyancy force and virtual mass force. $\mu_{l}$ is the liquid viscosity. The liquidphase volume fraction, $\alpha_{l}$ is defined as

$$
\alpha_{l}=1-\frac{\sum_{i} V_{\mathrm{d}, i}}{V_{\text {cell }}}
$$

where, $V_{\mathrm{d}, i}$ is the volume occupied by the discrete phases and $V_{\text {cell }}$ is the volume of the grid cell. The standard $k$ - $\varepsilon$ model is used to model turbulence. $\mu_{t}$ in Eq. (2) is the turbulent viscosity, which is defined as

$$
\mu_{t}=C_{\mu} \rho_{l} \frac{k^{2}}{\varepsilon}
$$

The standard $k-\varepsilon$ model is used to model turbulence, which means that the following transport equations of $k$ and $\varepsilon$ are solved.

$$
\begin{aligned}
& \alpha_{l} \rho_{l}\left(\frac{\partial k}{\partial t}+\vec{u}_{l} \cdot \nabla k\right)=-\nabla\left(\alpha_{l} \frac{\mu_{l}}{\sigma_{k}} \nabla k\right)+\alpha_{l} G_{k}-\alpha_{l} \rho_{l} \varepsilon \ldots \\
& \alpha_{l} \rho_{l}\left(\frac{\partial \varepsilon}{\partial t}+\vec{u}_{l} \cdot \nabla \varepsilon\right)=-\nabla\left(\alpha_{l} \frac{\mu_{l}}{\sigma_{\varepsilon}} \nabla \varepsilon\right)+\alpha_{l} C_{1} \frac{\varepsilon}{k} G_{k}-\alpha_{l} C_{2} \rho_{l} \frac{\varepsilon^{2}}{k} \\
& G_{k}=\mu_{t}\left(\frac{\partial u_{i, j}}{\partial x_{j}}+\frac{\partial u_{i, j}}{\partial x_{i}}\right) \frac{\partial u_{i, j}}{\partial x_{j}}
\end{aligned}
$$

The values of the constants are $C_{\mu}=0.09, \sigma_{k}=1.0, \sigma_{\varepsilon}=1.3$, $C_{1}=1.44, C_{2}=1.92$.

\subsection{Governing Equations for Discrete Phases}

The bubbles and inclusions are treated as discrete particles and their motion can be obtained by integrating the following transport equation for each particle, which considers contributions of six forces:

$$
m_{\mathrm{d}} \frac{d \vec{v}_{\mathrm{d}}}{d t}=\vec{F}_{\mathrm{d}}+\vec{F}_{\mathrm{p}}+\vec{F}_{\mathrm{b}}+\vec{F}_{\mathrm{vm}}+\vec{F}_{\mathrm{g}}+\vec{F}_{\mathrm{L}}
$$

The terms on the right-hand side of Eq. (8) are drag force, pressure gradient force, buoyancy force, virtual mass force, gravitational force, and lift force. Detail descriptions of these forces could be found in our previous works. ${ }^{16)}$ All forces are expressed as follows

$$
\begin{aligned}
& \vec{F}_{\mathrm{d}}=C_{\mathrm{d}} \frac{\rho_{\mathrm{f}}\left|\vec{v}-\vec{v}_{\mathrm{d}}\right|\left(\vec{v}-\vec{v}_{\mathrm{d}}\right)}{2} \frac{\pi d_{\mathrm{d}}^{2}}{4} \\
& \vec{F}_{\mathrm{p}}=\frac{1}{6} \pi d_{\mathrm{d}}^{3} \rho_{\mathrm{f}} \frac{d \vec{v}}{d t} \\
& \vec{F}_{\mathrm{b}}=-\frac{1}{6} \pi d_{\mathrm{d}}^{3} \rho_{\mathrm{f}} \cdot \vec{g} \\
& \vec{F}_{\mathrm{vm}}=\frac{1}{6} \pi d_{\mathrm{d}}^{3} C_{\mathrm{vm}} \rho_{\mathrm{f}} \frac{d}{d t}\left(\vec{v}-\vec{v}_{\mathrm{d}}\right) \\
& \vec{F}_{\mathrm{g}}=\frac{1}{6} \pi d_{\mathrm{d}}^{3} \rho_{\mathrm{d}} \cdot \vec{g}
\end{aligned}
$$




$$
\vec{F}_{\mathrm{L}}=-\frac{1}{6} \pi d_{\mathrm{d}}^{3} C_{\mathrm{L}} \rho_{\mathrm{f}}\left(\vec{v}_{\mathrm{d}}-\vec{v}\right)(\nabla \times \vec{v})
$$

The virtual mass coefficient $C_{\mathrm{vm}}$ and lift force coefficient $\mathrm{C}_{\mathrm{L}}{ }^{28)}$ are set as 0.5 for both spherical bubbles and solid particles. Bubbles and solid particles share one drag coefficient equation, ${ }^{29)}$ which depends on the flow regime and the liquid properties:

$$
\begin{aligned}
& C_{\mathrm{d}}=\frac{24}{R e_{\mathrm{d}}} \quad R e_{d}<2 \\
& C_{\mathrm{d}}=\frac{24}{R e_{\mathrm{d}}}\left(1+0.15 R e_{\mathrm{d}}^{0.687}\right) \quad 2<R e_{\mathrm{d}}<500 \\
& C_{\mathrm{d}} \approx 0.44 \quad R e_{\mathrm{d}}>500
\end{aligned}
$$

where $R e_{d}$ is the discrete particle Reynolds number and defined as $R e_{\mathrm{d}}=\frac{\rho_{f} d_{\mathrm{d}}\left|\vec{v}-\vec{v}_{\mathrm{d}}\right|}{\mu_{f}}$.

Random walk model was used to consider the effect of turbulent velocity fluctuation to the transport of particles. Lastly, the trajectories of discrete elements are determined by Eq. (18), as given below

$$
\vec{x}_{\mathrm{d}, i}=\int \vec{v}_{\mathrm{d}, i} d t
$$

\subsection{Interaction between Discrete Phases}

\subsubsection{Bubble Collision}

The liquid film between bubbles resists their relative motion when two bubbles are moving towards each other. As shown in Fig. 1, if the thickness of liquid film is larger than a critical value, bubbles will rebound; otherwise, bubbles will coalesce. A relative critical Weber number was defined by Eq. (19) as a criterion to differentiate these two conditions.

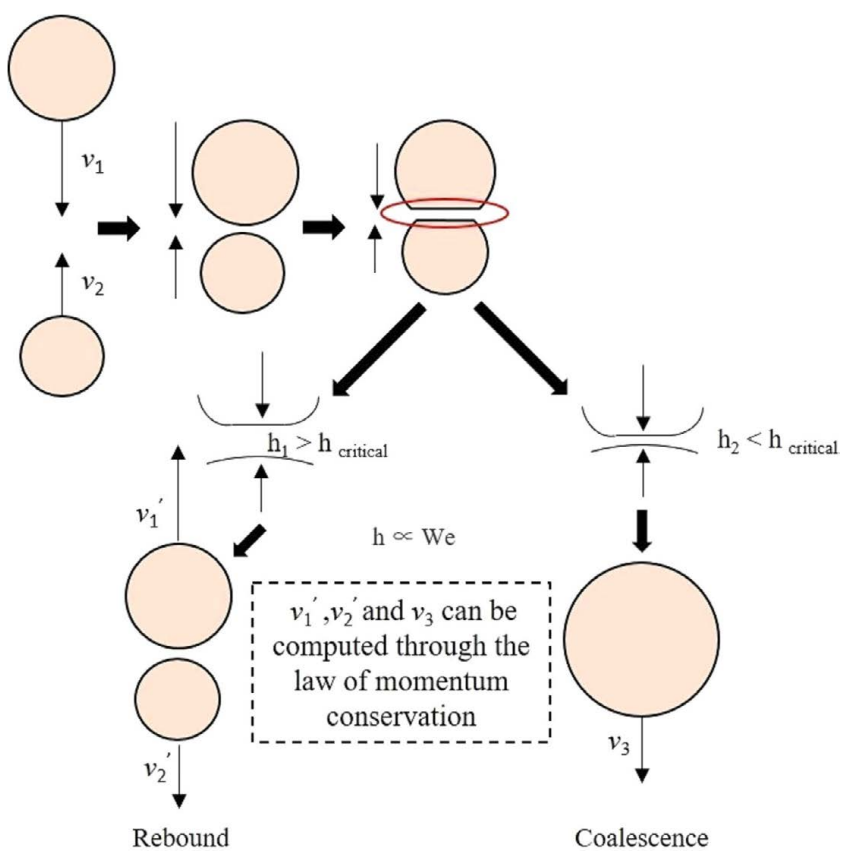

Fig. 1. The schematic of bubble collision. (Online version in color.)

$$
\mathrm{We}=\frac{d_{\mathrm{d}} v_{\mathrm{d}}^{\prime 2} \rho_{\mathrm{l}}}{\sigma}
$$

where $d_{\mathrm{d}}$ is the average diameter of two bubbles, $v_{\mathrm{d}}^{\prime}$ is the relative velocity, $\rho_{\mathrm{l}}$ is the liquid density and $\sigma$ is the liquid surface tension. There was discussion about this critical Weber number. Tsao and $\mathrm{Koch}^{30)}$ proposed a critical value of 1.6. But one of the bubbles was fixed. Duineveld ${ }^{31)}$ used the value of relative Weber number 0.18 for bubbles having short distances. Zhang et al. ${ }^{32)}$ used 0.14 as the value of the relative Weber number, and the bubbles were also close to others . This paper chose 0.16 as the relative critical Weber number based on the previous work. ${ }^{16)}$

When the relative Weber number is larger than the critical relative value, the approaching bubbles will rebound. In this work, the rebound of two bubbles is assumed to be elastic. Therefore, based on the law of momentum conversation, bubbles' velocities after bouncing can be obtained. When the relative Weber number is less than the critical relative value, two bubbles will coalesce. After coalescence, the new bubble's velocity $v_{3}$ is given by

$$
v_{3}=\frac{m_{1} v_{1}+m_{2} v_{2}}{m_{1}+m_{2}}
$$

Where $v_{1}, v_{2}$ are the velocities of two bubbles before interaction and $m_{1}, m_{2}$ are the mass of bubbles respectively.

Similarly, the velocity of the bubble after bouncing off can be obtained according the momentum conservation.

$$
\begin{aligned}
& v_{1}^{\prime}=2 \frac{m_{1} v_{1}+m_{2} v_{2}}{m_{1}+m_{2}}-v_{1} \\
& v_{2}^{\prime}=2 \frac{m_{1} v_{1}+m_{2} v_{2}}{m_{1}+m_{2}}-v_{2}
\end{aligned}
$$

where $v_{1}^{\prime}$ and $v_{2}^{\prime}$ are the velocities of two bubbles after rebound respectively.

\subsubsection{Bubble Breakup}

When the gas bubbles are in the turbulent region and therefore the fluctuation velocity gradient is large, the shear force produced in this process breaks bubbles into smaller ones. The turbulence intensity can be characterized by the turbulent kinetic energy dissipation rate. The bubble size decreases with the increase of turbulence intensity, and the maximum stable bubble size is fixed for a given fluid turbulence intensity, given by: ${ }^{33)}$

$$
d_{\mathrm{B}_{\max }} \approx W e_{\text {crit }}^{0.6}\left(\frac{\sigma \times 10^{3}}{\rho_{1} \times 10^{-3}}\right)(\varepsilon \times 10)^{-0.4} \times 10^{-2}
$$

where $d_{\mathrm{B}_{\max }}$ is the maximum stable bubble size; $\varepsilon$ is the turbulent kinetic energy dissipation rate; $\sigma$ is the surface tension; $\rho_{1}$ is liquid density; $W e_{\text {crit }}$ is the critical breakup Weber number. For argon-steel melt systems, $W e_{\text {crit }}=1.3,{ }^{33)}$ and $\sigma=1.192 .{ }^{9}$ ) When bubble size is larger than the maximum stable size, the bubble will break into two small ones with the same radius, as shown in Fig. 2. The process satisfies the momentum and mass conservation.

\subsubsection{Inclusions Attachment}

It was demonstrated ${ }^{20,21)}$ that almost all particles non- 


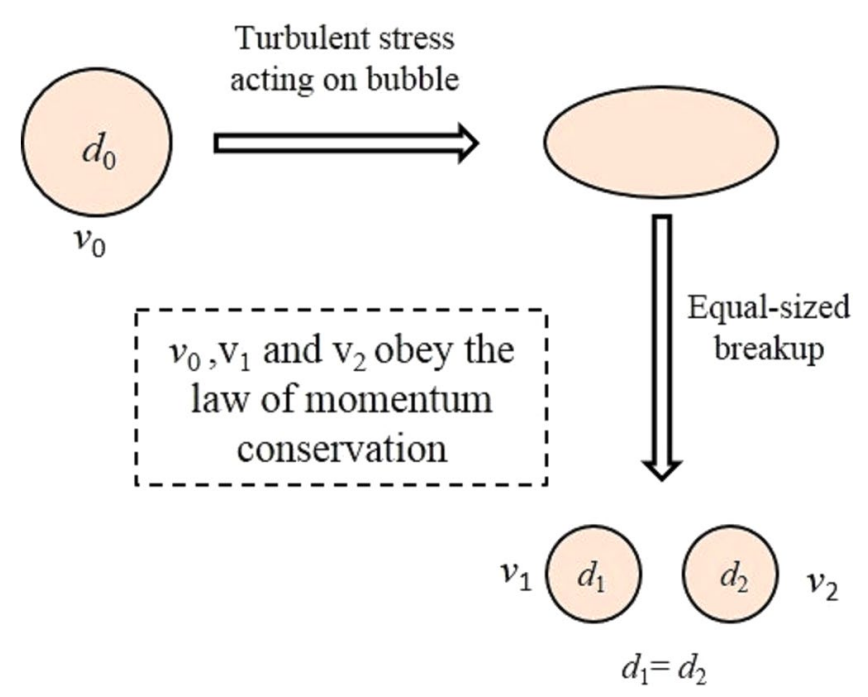

Fig. 2. The schematic diagram of bubble breakup. (Online version in color.)

wetting to the liquid phase will be attached to gas bubbles if they are close to a certain distance. This work assumes that all the alumina inclusions will attach to bubbles in bubbleinclusion collisions because the contact angle of alumina inclusion with liquid steel is $144^{\circ}$ (non-wetting).

Located in the trajectory of a bubble, inclusions may collide with the bubble or not, resulting from the flow around the bubble (Fig. 3). At present, this process could not be simulated directly when numerous bubbles are involved, due to the high demand of computer resources. Hence, particle-bubble collision models were used to consider this process, which was reviewed by Dai et al. ${ }^{18)}$ It shows that when stokes number is smaller than $1 / 12$, the influence of inertial forces can be neglected and the Sutherland model ${ }^{34)}$ is applicable to calculate the bubble-inclusion collision probability $p_{\mathrm{c}}$ as given by Eq. (24).

$$
p_{\mathrm{c}}=3 d_{\mathrm{i}} / d_{\mathrm{b}} \text {. }
$$

where $d_{\mathrm{i}}$ and $d_{\mathrm{b}}$ represent diameter of inclusion and bubble respectively. For the studied cases, the Stokes number is less than $1 / 12$ for inclusions smaller than $50 \mu \mathrm{m}$ in diameter and the Sutherland model is applicable. The process is thus simplified into the collision of two rigid spheres and the calculation of velocity follows the law of momentum conservation. If the inclusion is attached to the bubble, they will move together as a bubble-inclusion cluster with the total weight of the bubble and the attached inclusion. Specially, if a bubble-inclusion cluster encounter with an inclusion, it will also be treated as bubble-inclusion interaction in this model.

Concretely, in each timestep, distances between pairwise inclusions and bubbles are calculated. If their distance smaller than sum of radius and a random number (between 0 and 1) smaller than their collision probability, they will be treated as a bubble-inclusion cluster generated after a collision, whose parameters given by

$$
\begin{gathered}
m_{\mathrm{c}}=m_{\mathrm{i}}+m_{\mathrm{b}} \\
v_{\mathrm{c}}=\frac{m_{\mathrm{i}} v_{\mathrm{i}}+m_{\mathrm{b}} v_{\mathrm{b}}}{m_{\mathrm{i}}+m_{\mathrm{b}}}
\end{gathered}
$$

where subscript c, i and b represent the cluster, inclusion

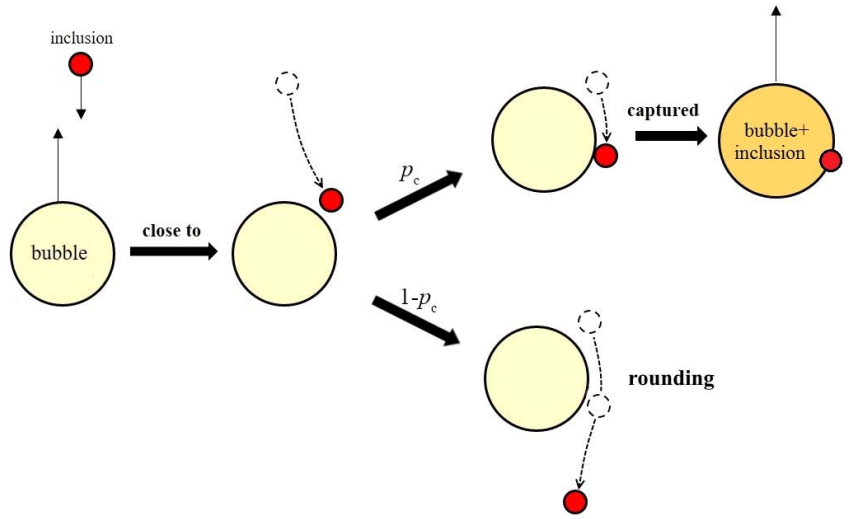

Fig. 3. The schematic diagram for bubble and inclusion attachment. (Online version in color.)

and bubble respectively. Because the size of the inclusions is much smaller than that of the bubbles, the volume and radius of cluster are assumed to be equal to those of the bubble. By tracking those bubble-inclusion clusters as discrete phases model aforementioned, locations and behaviors of inclusions attached to bubble could be investigated comprehensively.

\subsection{Assumptions}

In turbulent dispersion systems, the fluid dynamics and interaction of discrete phases are rather complex, and many resolved issues exist. Hence, a few assumptions are necessary to establish the current model.

1. It is assumed that bubbles will break into only two smaller ones. The experimental results of Heskett et $a l .{ }^{35)}$ support this assumption. They found that all bubbles breakage events were binary in turbulent pipeline flows. There are many controversies on the issue of daughter bubble size distribution. ${ }^{36,37)}$ Some researchers $^{38,39)}$ assume that equal size breakage is most likely, while others suppose unequal breakage. ${ }^{40)}$ Lehr et al. ${ }^{41)}$ proposed a model that combines previous studies in break of bubble and their simulation results calculated through this model are in good agreement with the bubble columns experiment. Their research indicates that the breakage into two equal sized bubbles is easiest for small bubbles. For the fluid density, surface tension and turbulent dissipation rate (within $0.08 \mathrm{~m}^{2} / \mathrm{s}^{3}$ ) in our simulation, Lehr's model shows that when the diameter of the mother bubble is less than $7.364 \mathrm{~mm}$, equal size breakage is most likely. In this work, the diameters of investigated bubbles are within this range, so the equal size breakup is assumed in our model.

2. The inclusions attached to the bubble are randomly distributed to two daughter bubbles in bubble breakup.

3. The effect of bubble wake on the inclusions is neglected.

4. The attached inclusions are assumed to be fixed on the surface of bubbles and their sliding processes and detachments are ignored.

5. Collision and coalescence between inclusions are not considered, as its mechanism is not clear and the probability of collision between small inclusions is very small. $^{42)}$ 
6. Bubbles and inclusions are assumed to be spherical. Their shapes and sizes do not change with temperature and pressure.

\section{Simulation Conditions}

The 3D turbulence flow of the fluid and the trajectories of gas bubbles and inclusions in a continuous casting mold (Fig. 4) were simulated. The equations are solved with the commercial package FLUENT with user defined subroutines for discrete phases and the interaction between the fluid phase and discrete phases. The geometrical and process parameters are given in Table 1. The model consists of 150000 cells and further grid refinement did not bring

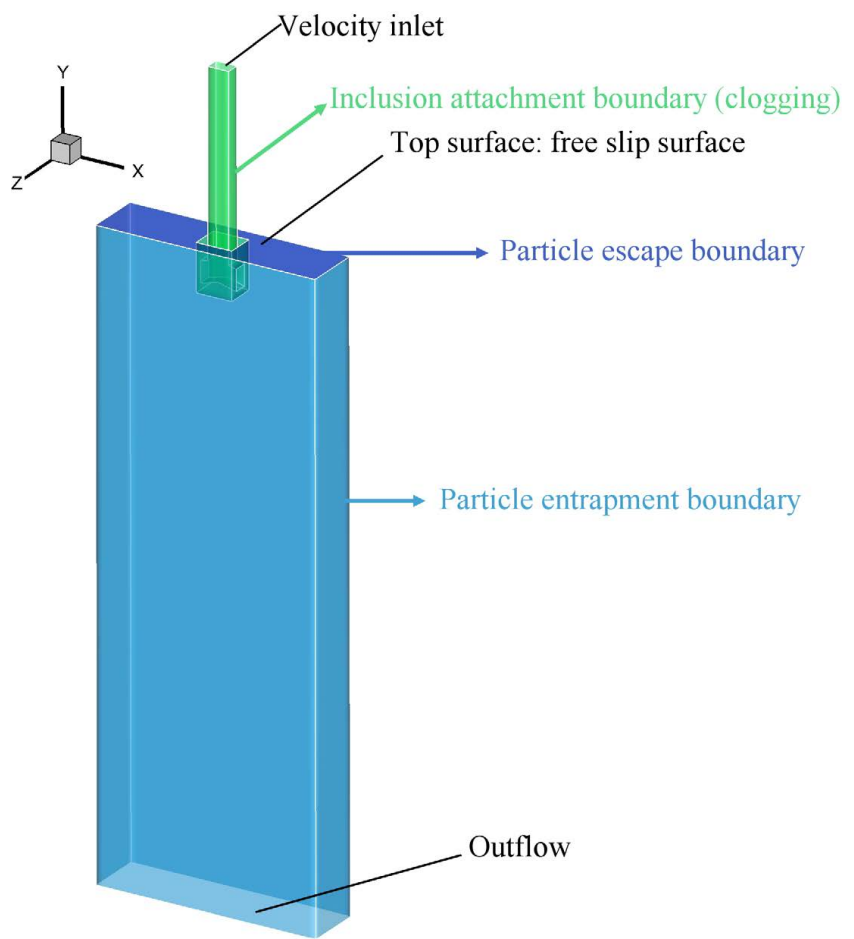

Fig. 4. Schematic diagram of the mold and boundary conditions. (Online version in color.)

Table 1. Geometrical and process parameters.

\begin{tabular}{lc}
\hline \multicolumn{1}{c}{ Parameter } & Value \\
\hline Slab width $(\mathrm{mm})$ & 420 \\
Slab thickness $(\mathrm{mm})$ & 108 \\
Slab length $(\mathrm{mm})$ & 1200 \\
SEN submerged depth $(\mathrm{mm})$ & 78 \\
Nozzle exit area $\left(\mathrm{mm}^{2}\right)$ & 711 \\
Casting speed $\left(\mathrm{m} / \mathrm{min}^{2}\right)$ & 1.5 \\
Fluid dynamic viscosity $\left(\mathrm{N} \cdot \mathrm{s} / \mathrm{m}^{2}\right)$ & 0.00555 \\
Fluid density $\left(\mathrm{kg} / \mathrm{m}^{3}\right)$ & 7020 \\
Gas-liquid surface tension $\left(\mathrm{N} / \mathrm{m}^{2}\right)$ & 1.192 \\
Gravity acceleration $\left(\mathrm{m} / \mathrm{s}^{2}\right)$ & 9.81 \\
Gas density $\left(\mathrm{kg} / \mathrm{m}^{3}\right)$ & 0.291 \\
Inclusion density $\left(\mathrm{kg} / \mathrm{m}^{3}\right)$ & 3600 \\
SEN port angle $(\mathrm{degree})$ & $15($ downward $)$ \\
\hline
\end{tabular}

significant changes to the simulation results with the standard $k$ - $\varepsilon$ turbulence model. The time step for the simulation is $0.001 \mathrm{~s}$.

For the molten steel, a constant velocity inlet boundary condition at the top of the SEN and an outflow outlet condition at the bottom of the calculation domain are applied. The top surface of the liquid being in contact with the slag is assumed to be flat. Here, a free-slip condition is used. The boundary condition between the molten steel and the SEN wall is no-slip while the SEN itself is considered to be stationary. Meanwhile, all the side walls of the liquid pool are assumed to be stationary and no-slip as well.

For the discrete phases (bubbles and inclusions), the escape boundary condition is defined for the mold top surface and the entrapment boundary condition for the side walls and outlet of mold. To take SEN clogging into consideration, the wall of SEN is modeled to be an attachment boundary for inclusions and reflect boundary for bubbles.

Inclusions flow into the mold with the molten steel at a uniform concentration from the nozzle inlet surface, while gas bubbles are injected from the wall of the SEN and enter the model through the two nozzle ports following the flow stream. Inclusion concentration in simulation is calculated from the number of inclusions added per second and the flow rate of molten steel. Number of injected bubble in unit time are determined by gas flow rate and initial bubble diameter. For the bubbles, a constant velocity inlet boundary condition is set at the wall of SEN. It is assumed that the bubbles remain spherical and their shape variations are neglected. The initial velocity is set as zero. To facilitate the analysis of the effect of bubbles on the removal of inclusions, the initial bubble size is set as constant in one case.

\section{Results and Discussion}

\subsection{Gas-liquid Two-phase Flow with Bubble Coales- cence and Breakup}

Before the discussion of interaction of bubbles and inclusions, properties of gas-liquid flow are analyzed briefly, whose details can be referred to our previous works. ${ }^{16,43)}$ In fixed gas flow rate of $2.7 \mathrm{~L} / \mathrm{min}$, cases are simulated to be stable under different initial bubble diameter. The size and spatial distribution of bubbles are plotted, and the distance between bubbles and SEN in the mold are calculated and plotted. As shown in Fig. 5, there are different sizes of bubbles in the mold under the specific initial bubble diameter, which is caused by coalescence and breakup. Meanwhile the size distribution of bubbles influences their trajectories, and thus affects their spatial distribution, whose details are as follows. The motion of bubbles can be divided into transverse motion and upward motion. The transverse motion is affected by the impact of the main flow of the molten steel along the outlet of the nozzle and the upward movement is affected by the floatation velocity of the bubble relative to the molten steel. The floatation speed of small bubbles is smaller than that of large bubbles, ${ }^{17)}$ so the motion of the small bubbles embodies the characteristics of transversely far away from the nozzle and the slow floatation, which have long trajectory. For large bubbles, the floating effect is the dominant factor with inadequate time to move transversely, so their trajectory is shorter, which is close 


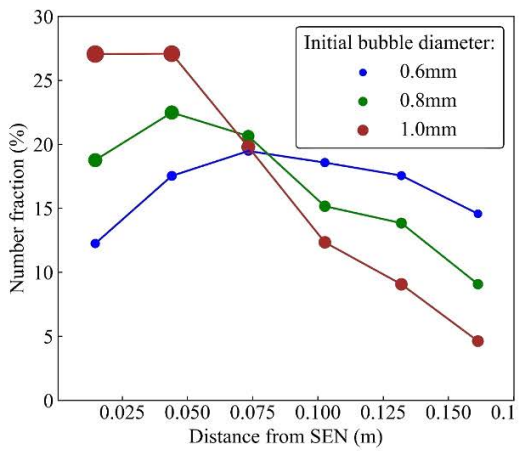

(a)

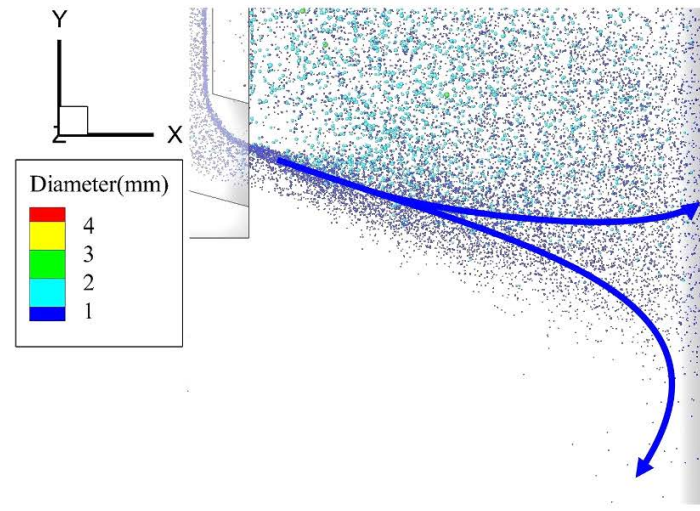

(b) $0.6 \mathrm{~mm}$

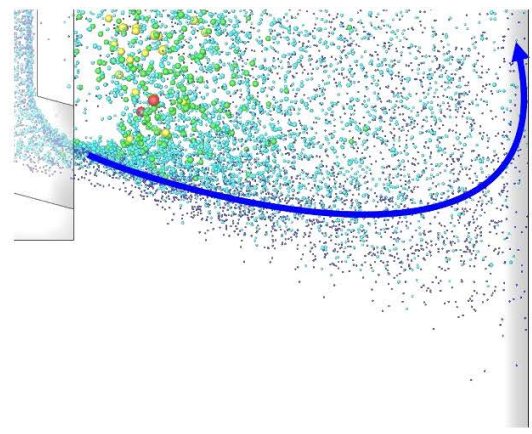

(c) $0.8 \mathrm{~mm}$

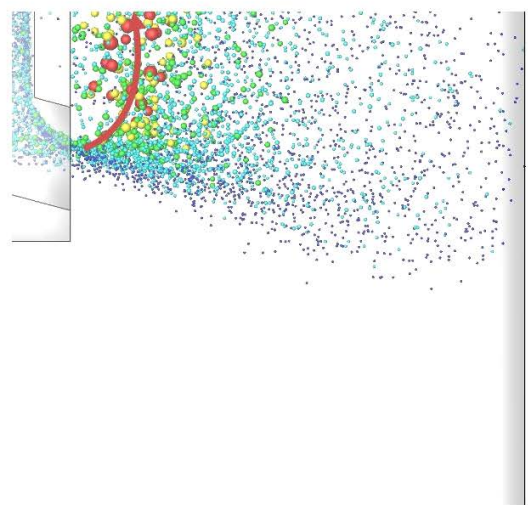

(d) $1.0 \mathrm{~mm}$

Fig. 5. Spatial distribution of bubbles for different initial bubble diameters $(0.6 \mathrm{~mm}, 0.8 \mathrm{~mm}$ and $1.0 \mathrm{~mm})$, where the gas flow rate is $2.7 \mathrm{~L} / \mathrm{min}$. (a) Sizes of points represent Sauter mean diameter of bubbles in an area. (b) $0.6 \mathrm{~mm}$. (c) $0.8 \mathrm{~mm}$. (d) $1.0 \mathrm{~mm}$. (Online version in color.)

to the immersion depth of the nozzle. As demonstrated by arrowed line in Fig. 5(d), driven by buoyancy, large bubbles float upward near the SEN and escape from the liquid steel surface. As shown in Fig. 5(c), following liquid steel stream (arrowed line), small bubbles tend to move toward sidewall and some of them that are close to sidewall could be entrapped. Bubbles less than $1.0 \mathrm{~mm}$ in diameter might follow the lower roll of flow and penetrate deeply as shown by lower arrowed line in Fig. 5(b).

As discussed above, the smaller the bubble is, the easier it is to be captured, so the initial bubble diameter is an important factor. Figure 5(a) is the statistics of bubble position in Figs. 5(b), 5(c), 5(d). It shows that the smaller initial bubble diameter, the greater the fraction of bubbles far away from SEN and close to sidewall, which means more bubbles may be entrapped by the sidewall. It is also confirmed by Fig. 6 that the smaller the initial bubble diameter is, the higher overall entrapment rate of bubbles becomes. When the initial bubble diameter is less than $1.0 \mathrm{~mm}$, the entrapment rate increases significantly, which cause harm that cannot be ignored.

\subsection{Relationship between Bubble Distribution and Inclusion Behaviors}

In molten steel, the density of inclusions is very close to the that of molten steel, so their relative velocities to molten steel are nearly zero and they mainly move with the surrounding fluid to the solidification front. For small bubble the final rising speed is lower than that of large bubble, ${ }^{17)}$ thus motion characters of small bubble in molten steel are closer to that of inclusions. As shown in Fig. 7, under the

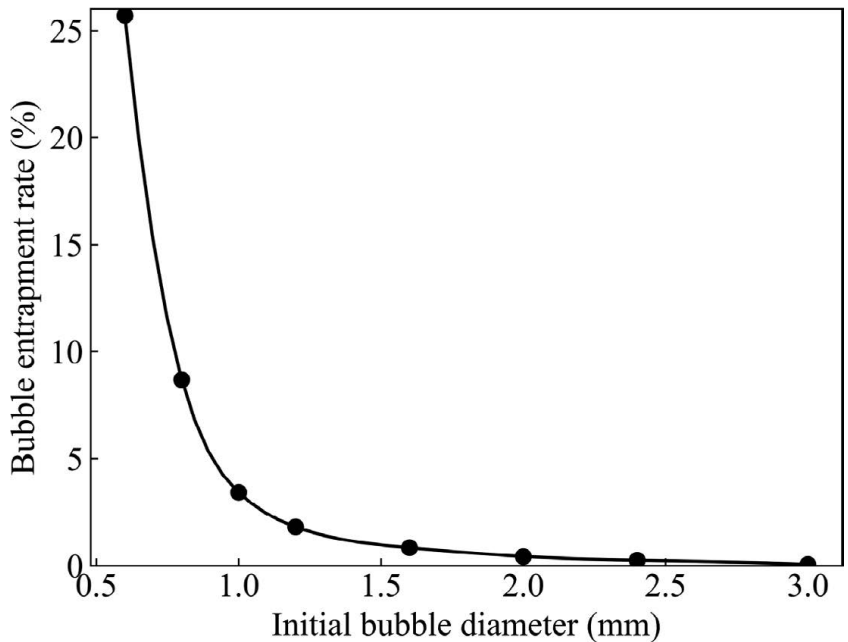

Fig. 6. Bubble entrapment rate for different initial bubble diameters.

aforementioned factors, small bubbles (line 2) are closer to the trajectories of inclusions (line 3), while large bubbles tend to float upward (line 1) and be separated to the trajectories of inclusions after leaving the nozzle. The inclusionbubble attachment changes the total weight of bubble. So, instead of argon density, apparent density is used to measure density of bubble, calculated by

$$
\rho_{\text {apparent }}=\frac{\rho_{\text {argon }} V_{\mathrm{b}}+\sum_{j} \rho_{\mathrm{i}} V_{\mathrm{i}, j}}{V_{\mathrm{b}}}
$$

where $\mathrm{j}$ is the serial number of inclusions attached to spe- 


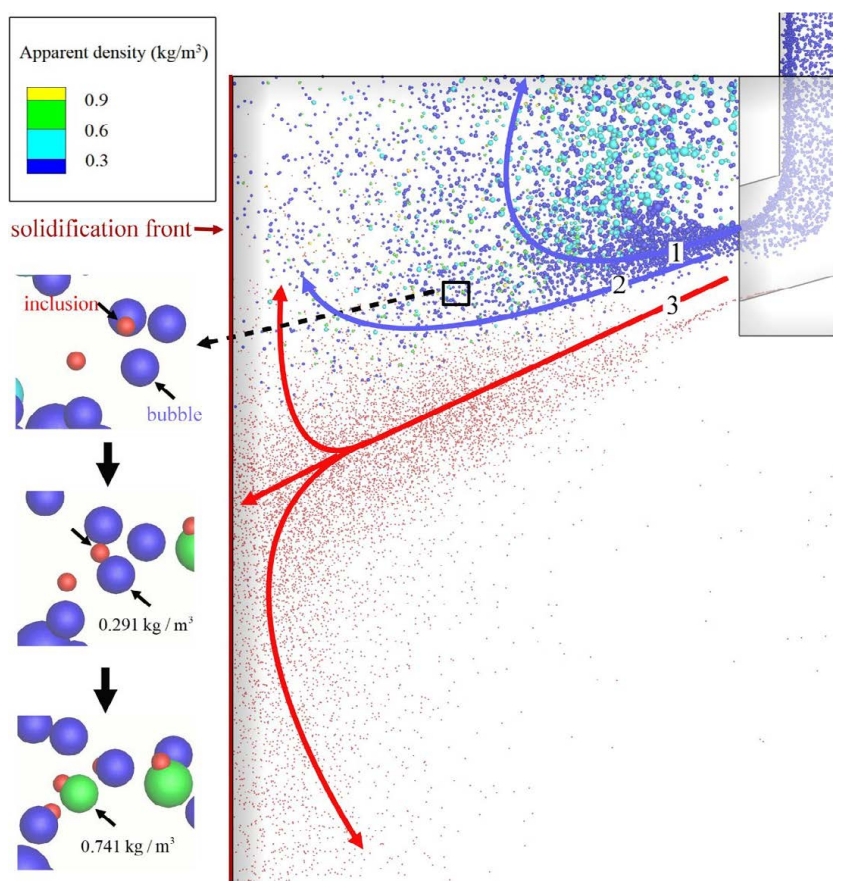

Fig. 7. Transport and interaction of bubbles and inclusions (inclusions' diameter are magnified 5 times). (Online version in color.)

cific bubble. Because the size of the inclusions is much smaller than that of the bubbles, it is assumed that attached inclusions do not change the volume of bubble. The origin density of argon bubble in the high temperature molten steel $(1793 \mathrm{~K})$ is $0.291 \mathrm{~kg} / \mathrm{m}^{3}$ and the more inclusions a bubble absorbs, the higher apparent density it has. Local amplification of Fig. 7 shows that the density of a bubble increases after its colliding and absorbing an inclusion.

20000 inclusions are injected in the first two seconds, while argon bubbles are continuously injected for $20 \mathrm{~s}$. After simulation for $20 \mathrm{~s}$, most inclusions are either removed or entrapped and few remain in molten steel. Some of them are independent inclusion behaviors, the others are behaviors after attachment to bubble. For instance, some inclusions are removed by independently floating and others are carried and removed by bubble. In order to distinguish these behaviors, trace and count the various situations of inclusions after simulating for $20 \mathrm{~s}$, and calculate the proportions of various behaviors, which constitute one column of Fig. 8. The behaviors of inclusions are investigated under different initial bubble diameters. The trends of 'Entrapment by attachment to bubble' and 'Removal by attachment to bubble' in Fig. 8 show a mentionable phenomenon. That is, the smaller the initial bubble, the more inclusions adhered to the bubble, because smaller bubbles have longer trajectory as explained in section 4.2 and also supported by the bubble path length statistics, ${ }^{23)}$ higher probability of collision with inclusions (Eq. (24)), larger total surface area under same gas flow rate and closer trajectories with inclusions (Fig. 7).

In most situation, the 'bubble + inclusion' will float upward and be removed into top slag. However, when the initial bubble diameter is less than $1.0 \mathrm{~mm}$, some of the inclusions adhering to the bubbles are captured by solidifying shell (Fig. 8), resulting from high entrapment rate of small bubbles. As the initial bubble diameter decreases,

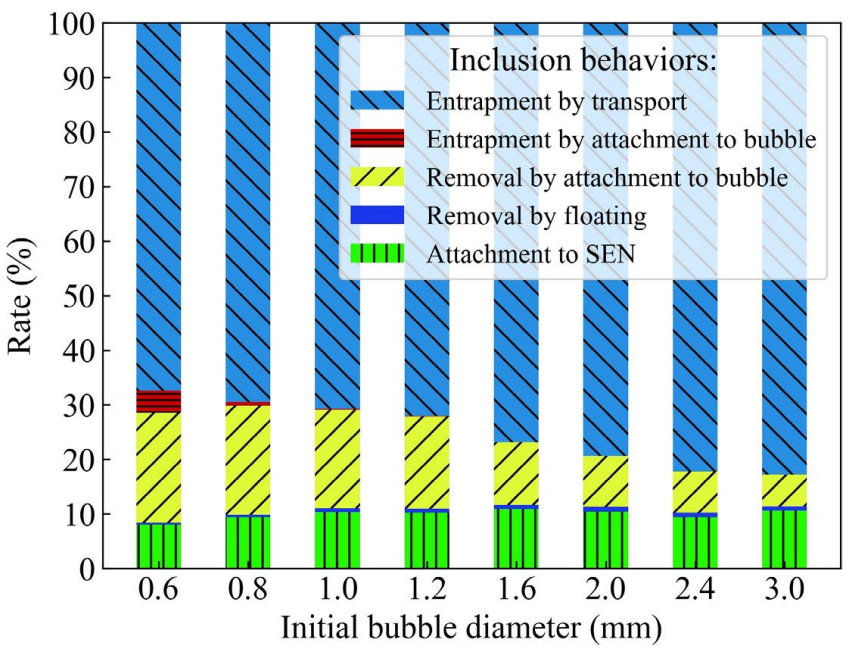

Fig. 8. Fractions of inclusion behaviors. (Online version in color.)

more and more 'bubbles + inclusions' are captured, which causes not only decreases of inclusion removal rate but also serious harm to steel products due to special property of 'bubble + inclusion'. ${ }^{2)}$ Considering all the factors, the optimal initial bubble diameter should be around $1.0 \mathrm{~mm}$.

Overall, smaller bubbles are more capable of removing inclusions. However, excessive small bubbles cause more serious defects, which is supposed by some researchers ${ }^{2,23}$ ) but has not been simulated or quantified due to the limitations of traditional model. The optimal initial bubble diameter is $1.0 \mathrm{~mm}$. And, more importantly, not all inclusions attached to bubbles will be removed, so their subsequent behaviors should be taken seriously.

\subsection{Removal Rate of Inclusions in Various Conditions}

In simulations of this section, 20000 inclusions are injected in the first two seconds, while argon bubbles are continuously injected in $20 \mathrm{~s}$. The removal rates of inclusions are investigated under different conditions.

Different to bubble removal, it is difficult to remove small inclusions from the mold only by their own floatation for their large density and consequent low upward velocity. While blowing argon is an effective way to remove inclusions. As shown in Fig. 9, without argon injection, the removal rate of inclusions is only about $10 \%$, while with argon injection, it rises with the increase of argon gas flow rate significantly and reach almost $30 \%$ under gas flow rate of $2.7 \mathrm{~L} / \mathrm{min}$. In all, bubble adhesion removal is the main way for inclusion removal. Researches of the removal of inclusions in the continuous casting without considering bubble-inclusion attachment will get results with huge errors.

As shown in Fig. 10, in most situation, larger inclusions have a higher removal rate, which agrees well with previous studies. Overall, the removal rates are from $14 \%$ to $30 \%$, which are roughly consistent with the result (Inclusions decrease $22 \%$ from tundish to slab) of industrial experiment carried by Zhang et al. ${ }^{44)}$ The main deviation between simulation prediction and experiment comes from the uncertainty of the actual initial bubble size in the production process and the single inclusion size distribution used in the simulation for the limitation of the model and the computation cost. If the initial bubble size in the simulation is set to $1 \mathrm{~mm}$ 


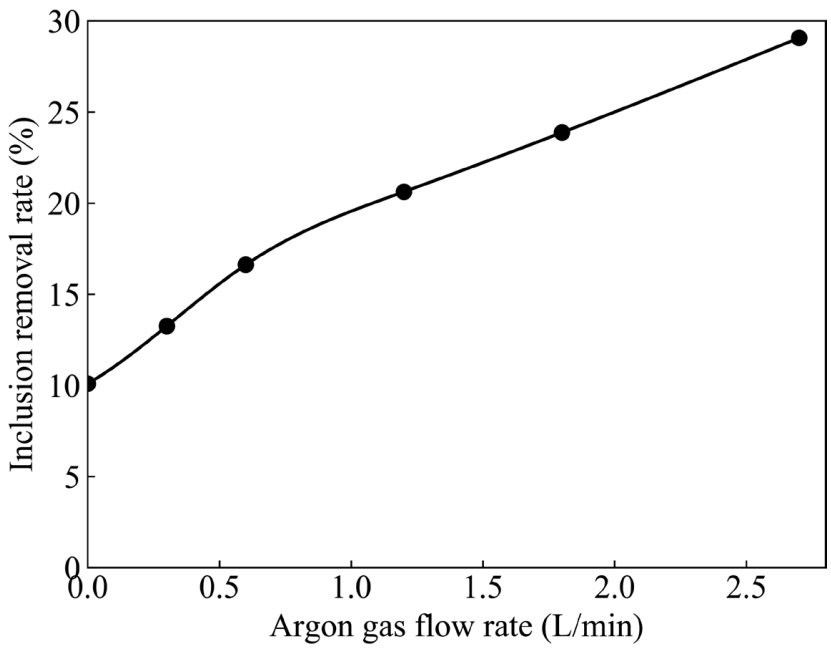

Fig. 9. Inclusion removal rate under different argon gas flow rates. Initial bubble diameter is $1.0 \mathrm{~mm}$ and inclusion diameter is $50 \mu \mathrm{m}$

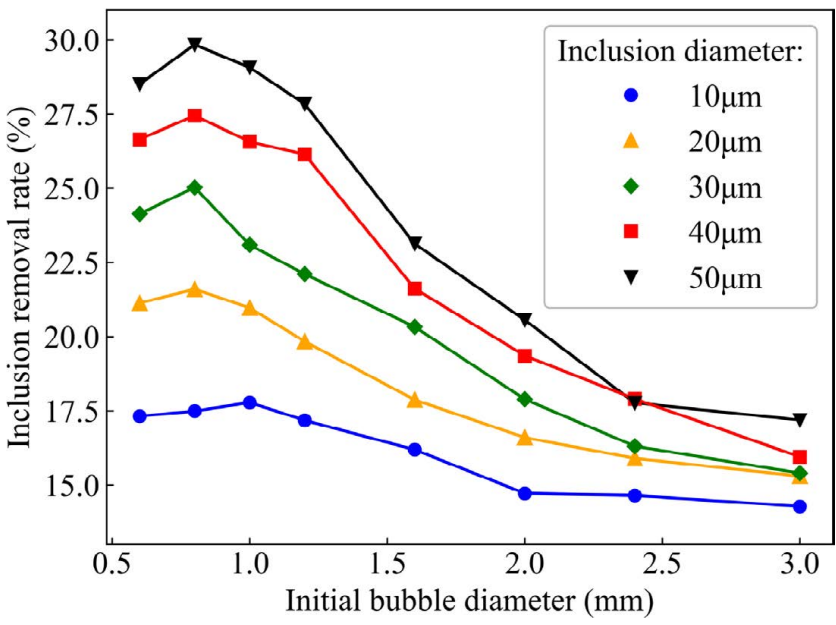

Fig. 10. Inclusion removal rate at different initial bubble diameters and inclusion diameters where the gas flow rate is 2.7 $\mathrm{L} / \mathrm{min}$. (Online version in color.)

used by Liu et al. ${ }^{13)}$ and the analysis object is restricted to inclusions around $20 \mu \mathrm{m}$ with highest ppm content in the experiment, the removal rate in simulation is about $21 \%$ and the agreement with the experimental result of $22 \%$ is much better. To carry out further research, the improvement of simulation models and experimental bubble detection methods are necessary. Under the constant gas flow rate of $2.7 \mathrm{~L} / \mathrm{min}$, the smaller the initial bubble, the higher the inclusion removal rate, which is agree with previous studies. ${ }^{23)}$ However, When the initial bubble diameter is less than $0.8 \mathrm{~mm}$, it shows an opposite trend. Because when a bubble is small enough, it along with attached inclusions may move to the solidifying front and be captured, instead of floating upward, as mentioned before.

\section{Conclusions}

A numerical model with detailed consideration of interactions between the discrete phases (bubbles and inclusions) is developed to investigate the effect of bubbles-inclusion attachment on the motion and removal of inclusions under various conditions. The predicted removal rates are com- pared with experimental measurements and good agreement is observed. The new findings are summarized below.

In previous researches, all inclusions attached to bubbles will be removed, which lead to a result that under constant gas flow rate, the smaller bubbles are, the higher total inclusion removal rate is. However, in this paper, when the initial bubble diameter is less than $0.8 \mathrm{~mm}$, it shows an opposite trend due to high entrapment rate of small bubbles attached with inclusions. This new result is attributed to the application of the new model, in which the subsequent behaviors of inclusions attached to bubbles are considered instead of being simplified to purely removal as traditional model, and bubble size distribution caused by the coalescence and breakup are calculated instead of uniform bubble sizes in previous studies.

The essential cause for this new result is the phenomenon that large bubbles float upward near SEN and escape from the liquid steel surface, while small bubbles tend to move toward sidewall while floating or even follow lower roll of flow and penetrate deeply. Some of those small bubbles are entrapped by solidifying shell, and the entrapment of bubbles attached with inclusions cause more serious defects and low inclusions removal rate, which is supposed by some researchers but has not been simulated or quantified due to the limitations of traditional model.

Bubble attachment removal is the main mode of mold inclusion removal. So, it will bring great deviation if this mode is ignored. Larger inclusions have a higher removal rate and the removal rates are from $14 \%$ to $30 \%$ under different initial bubble diameters, which are not high and agree roughly well with the result of previous industrial experiment. Combining the above conclusions, in practical production, decreasing bubble size is an effective way to promote inclusion removal and excessively small bubble should be avoided. Under the condition of this paper, the optimum initial bubble size is $1.0 \mathrm{~mm}$.

\section{Nomenclature}

$C_{\mathrm{d}}:$ drag force coefficient

$C_{\mathrm{vm}}$ : virtual mass force coefficient

$C_{\mathrm{L}}$ : lift force coefficient

$\mathrm{C}_{\mu}, C_{1}, C_{2}$ : standard k- $\varepsilon$ model constant

$d$ : diameter $(\mathrm{m})$

$F_{\mathrm{b}}$ : buoyancy force $(\mathrm{N})$

$F_{\mathrm{d}}$ : drag force $(\mathrm{N})$

$F_{\mathrm{g}}$ : gravitational force $(\mathrm{N})$

$F_{\mathrm{L}}$ : lift force $(\mathrm{N})$

$F_{\mathrm{p}}$ : pressure gradient force $(\mathrm{N})$

$F_{\mathrm{vm}}$ : virtual mass force $(\mathrm{N})$

$G_{k}$ : generation of turbulence kinetic energy due to the mean velocity gradients $\left(\mathrm{kg} \mathrm{m}^{-2} \mathrm{~s}^{-3}\right)$

$\mathrm{g}$ : acceleration of gravity $\left(\mathrm{N} \mathrm{kg}^{-1}\right)$

$m$ : mass $(\mathrm{kg})$

$p$ : static pressure $(\mathrm{Pa})$

$v$ : velocity $\left(\mathrm{m} \mathrm{s}^{-1}\right)$

$V_{\text {cell: }}$ grid cell volume $\left(\mathrm{m}^{3}\right)$

$V_{\mathrm{d}, i}$ : volume of $i$ th discrete particle $\left(\mathrm{m}^{3}\right)$

$W e$ : Weber number

Greek letters

$\alpha$ : phase fraction

$\varepsilon$ : turbulent kinetic energy dissipation rate $\left(\mathrm{m}^{2} \mathrm{~s}^{-3}\right)$ 
Subscripts

$$
\begin{aligned}
& \mu: \text { viscosity }(\mathrm{Pa} \mathrm{s}) \\
& \rho: \text { density }\left(\mathrm{kg} \mathrm{m}^{-3}\right) \\
& \sigma: \text { surface tension }\left(\mathrm{N} \mathrm{m}^{-1}\right)
\end{aligned}
$$

b: bubble

c: bubble-inclusion cluster

$\mathrm{d}$ : discrete particle (including discrete bubble, inclusion and bubble-inclusion cluster)

i: inclusion

1: liquid (molten steel in this paper)

\section{REFERENCES}

1) B. G. Thomas, Q. Yuan, S. Mahmood, R. Liu and R. Chaudhary: Metall. Mater. Trans. B, 45 (2013), 22.

2) Y. Miki and S. Takeuchi: ISIJ Int., 43 (2003), 1548.

3) H. Pan, X. Z. Chen, X. F. Liang, L. T. Zhu and Z. H. Luo: Powder Technol., 299 (2016), 235.

4) S. Sundaresan: AlChE J., 46 (2000), 1102.

5) K. Jin, B. G. Thomas and X. M. Ruan: Metall. Mater. Trans. B, 47 (2015), 548.

6) Z. Q. Liu, B. K. Li and M. F. Jiang: Metall. Mater. Trans. B, 45 (2013), 675.

7) L. F. Zhang, Y. F. Wang and X. J. Zuo: Metall. Mater. Trans. B, 39 (2008), 534.

8) H. Lei, Y. L. Jin, M. Y. Zhu and J. C. He: J. Mater. Sci. Technol., 18 (2002), 403

9) H. Bai and B. G. Thomas: Metall. Mater. Trans. B, 32 (2001), 1143

10) G. G. Lee, B. G. Thomas and S. H. Kim: Met. Mater. Int., 16 (2010), 501.

11) A. Ramos-Banderas, R. D. Morales, R. Sanchez-Perez, L. GarciaDemedices and G. Solorio-Diaz: Int. J. Multiph. Flow, 31 (2005), 643.

12) Y. Kwon, J. Zhang and H. G. Lee: ISIJ Int., 46 (2006), 257.

$13)$ Z. Q. Liu, L. M. Li, F. S. Qi, B. K. Li, M. F. Jiang and F. Tsukihashi: Metall. Mater. Trans. B, 46 (2014), 406.

14) R. Sanchez-Perez, R. D. Morales, L. Garcia-Demedices, J. P. Ramos and M. Diaz-Cruz: Metall. Mater. Trans. B, 35 (2004), 85.

15) Q. Yuan, T. Shi, S. P. Vanka and B. G. Thomas: Minerals and Metals Processing, TMS, Warrendale, PA, (2002), 18.
16) T. Zhang, Z. G. Luo, C. L. Liu, H. Zhou and Z. S. Zou: Powder Technol., 273 (2015), 154

17) L. F. Zhang and S. Taniguchi: Int. Mater. Rev., 45 (2000), 59.

18) Z. F. Dai, D. Fornasiero and J. Ralston: Adv. Colloid Interface Sci., 85 (2000), 231.

19) Z. F. Dai, S. Dukhin, D. Fornasiero and J. Ralston: J. Colloid Interface Sci., 197 (1998), 275.

20) W. Pan, K. Uemura and S. Koyama: Tetsu-to-Hagané, 78 (1992), 1361.

21) X. F. Zheng, P. C. Hayes and H. G. Lee: ISIJ Int., 37 (1997), 1091.

22) L. H. Wang, H. G. Lee and P. Hayes: ISIJ Int., 36 (1996), 7.

23) L. F. Zhang, J. Aoki and B. G. Thomas: Metall. Mater. Trans. B, 37 (2006), 361.

24) L. T. Wang, Q. Y. Zhang, Z. B. Li and Z. L. Xue: J. Iron Steel Res. Int., 11 (2004), 5.

$25)$ Y. Kwon, J. Zhang and H. G. Lee: ISIJ Int., 48 (2008), 891

26) D. Q. Geng, H. Lei and J. C. He: ISIJ Int., 50 (2010), 1597.

27) W. T. Lou and M. Y. Zhu: Metall. Mater. Trans. B, 44 (2013), 762.

28) T. R. Auton: J. Fluid Mech., 183 (2006), 199.

29) A. Tomiyama: Multiph. Sci. Technol., 10 (1998), 369.

30) H. K. Tsao and D. L. Koch: Phys. Fluids, 9 (1997), 44.

31) P. C. Duineveld: Ph.D. thesis, Twente University, (1994), (https://www. researchgate.net/publication/35956628 Bouncing and Coalescence of Two Bubbles in Water), (accessed 2017-4-17).

32) X. Y. Zhang and G. Ahmadi: Chem. Eng. Sci., 60 (2005), 5089.

33) M. Sevik and S. H. Park: J. Fluids Eng., 95 (1973), 53.

34) K. L. Sutherland: J. Phys. Chem., 52 (1948), 394.

35) R. P. Hesketh, A. W. Etchells and T. W. F. Russell: Chem. Eng. Sci., 46 (1991), 1.

36) P. Chen, J. Sanyal and M. P. Dudukovic: Chem. Eng. Sci., 60 (2005), 1085.

37) Y. X. Liao and D. Lucas: Chem. Eng. Sci., 64 (2009), 3389.

$38)$ C. Martinez-Bazan, J. L. Montanes and J. C. Lasheras: J. Fluid Mech., 401 (1999), 183.

39) C. H. Lee, L. E. Erickson and L. A. Glasgow: Chem. Eng. Commun., 61 (1987), 181.

40) H. Luo and H. F. Svendsen: AIChE J., 42 (1996), 1225.

41) F. Lehr, M. Millies and D. Mewes: AIChE J., 48 (2002), 2426

42) Y. Kang, B. Sahebkar, P. R. Scheller, K. Morita and D. Sichen: Metall. Mater. Trans. B, 42 (2011), 522.

43) T. Zhang, Z. G. Luo, H. Zhou, B. Ni and Z. S. Zou: ISIJ Int., 56 (2016), 116

44) L. F. Zhang, B. G. Thomas, K. K. Cai, J. Cui and L. X. Zhu: ISS Tech 2003, ISS-AIME, Warrendale, PA, (2003), 141. 